\title{
FERTILITY SPARING TREATMENT WITH LEVONORGESTREL INTRAUTERINE DEVICE AFTER COMPLETE MACROSCOPIC HYSTEROSCOPIC RESECTION FOR WELL DIFFERENTIATED EARLY STAGE ENDOMETRIOD ADENOCARCINOMA IN YOUNG WOMEN
}

\author{
O.Kaabia, G. Nourallah, Z. Rym, L. Aymen, S. Aymen, B. Mohamed, K. Hédi . \\ Université de Sousse- Faculté de Médecine de Sousse- Hopital Farhat Hached- \\ LR12ES03- 4000- Sousse- Tunisia
}

\section{Introduction :}

Endometrial cancer (EC) is a common gynecologic malignancy in developing countries in menopausal women but its prevalence is low in young pre-menopausal women.

While hysterectomy with bilateral salpingo-oophorectomy is the standard initial treatment for stage IA endometrioid well differenciated adenocarcinoma, younger women may desire fertility sparing options. A local hormonal therapy with Levonorgestrel has been supported by many basic and experimental sudies.

In this study, we aim to evaluate the efficiency of levonorgestrelintrauterine device (LNG-IUD) as an adjuvant local hormonal therapy treatment after complete macroscopic hysteroscopic resection of well differenciated early-stage endometrioid carcinoma (EC) in young women (under 45) who wished to preserve their fertility.

\section{Methods:}

We conducted a retrospective study from a prospective monocentric database from January 2008 to January 2019 in the department of gynecology and obstetrics in Farhat Hached Teaching Hospital Sousse, Tunisia. We collected then analysed the data of all the patients under 45 year old with grade 1 endometrioid adenocarcinoma confined to the endometrium who were treated with LNG-IUD after complete macroscopic hysteroscopic resection. At 6 months of treatment, the histologic change of the endometrial tissue was assessed by both vaginal ultrasound and hysteroscopy with curettage. The regression rate at 6 months treatment was evaluated.

\section{Results:}

From a cohort of 226 patients with endometrial cancer diagnosed at our department during the 11 years of the study, 22 were under 45 year old of whom nine patients were FIGO Stage IA grade 1 endometroid adenocarcinoma.

These nine patients were included in our study.

Two were excluded because they were pregnant at the time of diagnosis of the cancer. In these two pregnant women, our strategy after a multidisciplinary panel discussion and according to the couples wish, the pregnancy was not terminated and closely followed with pelvic MRI to monitor any recurrency. The delivery with caesarean section was programmed at 35 weeks of amenorrhea followed by a radical hysterectomy with bilateral oophorectomy.

The 9 patients who have completed the treatment protocol, had to have a close follow up with endovaginal ultrasound and MRI every 3 months and a Hysteroscopy with biopsies every 6 months. The complete regression (CR) rate at 6 months was 33.3\% (3/9). There were 2 cases of progressive disease. The other 4 cases had positive biopsies with no evidence of endometrial tumor neither on the ultrasound nor on the MRI. Five patients reported some spotting as a treatment-related complication.

\section{Conclusion :}

The need for a fertility spearing treatment to early stage grade 1 endometrioid carcinoma in young women is real but not so frequent in our daily practice. LNG-IUD treatment as an adjuvant therapy to a complete macroscopic hysteroscopic resection has modest results .

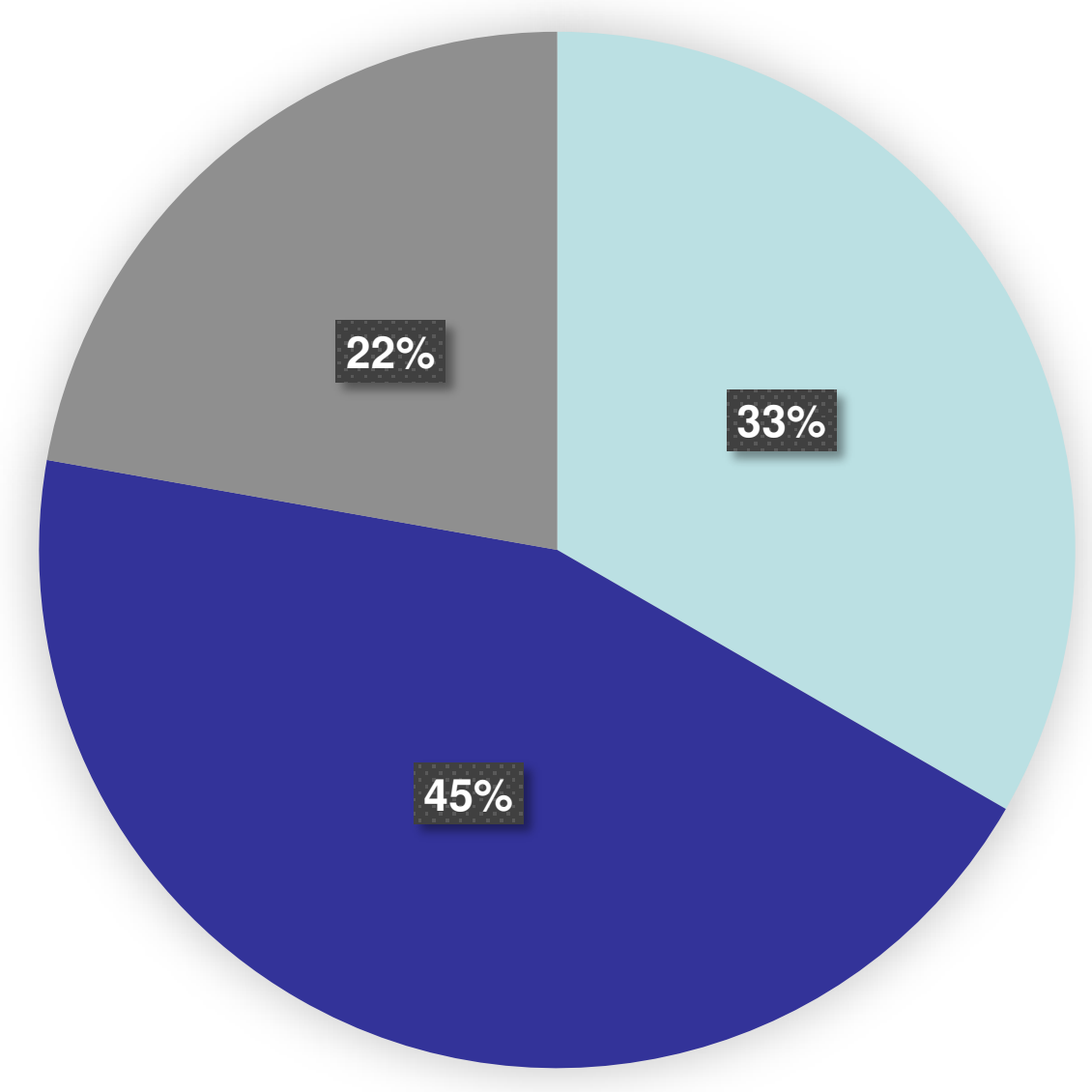

complete

regression

stable

Figure 1:

Disease progression

progression

disease 